\title{
Educação em saúde na Atenção Primária no ciclo gravídico puerperal: Uma revisão
}

\section{integrativa}

\author{
Health education in Primary Care during pregnancy-puerperium cycle: An integrative review \\ La educación sanitaria en Atención Primaria en el ciclo gravídico-puerperal: Una revisión \\ integradora
}

Recebido: 05/04/2021 | Revisado: 12/04/2021 | Aceito: 18/04/2021 | Publicado: 03/05/2021

Letícia Miná de Britto Cavalcanti ORCID: https://orcid.org/0000-0001-7647-7815 Centro Universitário de Patos, Brasil E-mail: leticiamina12@hotmail.com

Milena Nunes Alves de Sousa

ORCID: https://orcid.org/0000-0001-8327-9147 Centro Universitário de Patos, Brasil

E-mail: milenanunes@fiponline.edu.br

\begin{abstract}
Resumo
Introdução: A gestação e puerpério apresentam inúmeras transformações na vida da mulher. Nesse sentido, ações de educação em saúde são fundamentais para a prevenção de agravos, doenças e complicações. Objetivo: Analisar as ações de educação em saúde desenvolvidas na atenção primária para a prevenção de agravos e melhoria da qualidade de vida no ciclo gravídico puerperal. Método: Revisão integrativa da literatura a partir da biblioteca Scientific Electronic Library Online e base de dados Literatura Latino-Americana e do Caribe em Ciências da Saúde, Base de Dados de Enfermagem e U. S. National Library of Medicine, de 2016 a 2021. Utilizaram-se os seguintes Descritores em Ciências da Saúde e suas combinações nas línguas portuguesa e inglesa: "educação em saúde" AND "atenção primária" AND "gestantes"; "health education" and "primary care" and "pregnancy". A amostra final constituiu-se de 20 artigos. Resultados: Verificou-se que o período de maior abordagem foi no período de gestação contemplando 75\% dos artigos. Por fim, 75\% dos artigos estudados dizem respeito a práticas educativas, das quais a de maior destaque foi o diálogo, com 25\%. Entretanto, também se destacaram as oficinas educativas com 20\%. Conclusão: É notória a importância da abordagem da educação em saúde na atenção primária para qualificação da saúde da gestante e puérpera atribuída à prevenção de agravos, doenças e complicações nesse período. Sendo assim, destaca-se a necessidade de continuidade, intensificação e ampliação das estratégias educativas em saúde desenvolvidas na atenção primária como instrumento fundamental no ciclo gravídico puerperal.
\end{abstract}

Palavras-chave: Ciclo gravídico puerperal; Educação em saúde; Atenção Primária.

\begin{abstract}
Introduction: Pregnancy and puerperium present countless transformations in women's lives. In this sense, health education actions are essential for the prevention of injuries, diseases and complications. Objective: To analyze the actions of health education developed in primary care for the prevention of diseases and improvement of quality of life in pregnancy-puerperium cycle. Method: Integrative literature review from the Scientific Electronic Library Online and Latin American and Caribbean Literature on Health Sciences, Nursing Database and U. S. National Library of Medicine databases, from 2016 to 2021. The following Health Sciences Descriptors and their combinations in Portuguese and English were used: "health education" AND "primary care" AND "pregnant women"; "health education" and "primary care" and "pregnancy". The final sample consisted of 20 articles. Results: It was found that the period of greatest approach was during pregnancy, with $75 \%$ of the articles. Finally, $75 \%$ of the articles studied were related to educational practices, of which dialogue was the most prominent, with $25 \%$. However, educational workshops also stood out with $20 \%$. Conclusion: It is notorious the importance of the approach to health education in primary care for the qualification of the health of pregnant and postpartum women attributed to the prevention of injuries, diseases and complications in this period. Thus, it highlights the need for continuity, intensification and expansion of educational strategies in health developed in primary care as a fundamental tool in the gravidic-puerperal cycle.
\end{abstract}

Keywords: Pregnancy-puerperal cycle; Health Education; Primary Health Care.

\section{Resumen}

Introducción: La gestación y el puerperio presentan múltiples transformaciones en la vida de la mujer. En este sentido, las acciones de educación sanitaria son esenciales para la prevención de lesiones, enfermedades y complicaciones. 
Objetivo: Analizar las acciones de educación en salud desarrolladas en la atención primaria para la prevención de los trastornos y la mejora de la calidad de vida en el ciclo gravídico puerperal. Método: Revisión de literatura integrativa de la Biblioteca Electrónica Científica en Línea biblioteca y base de datos de Literatura Latinoamericana y Caribeña sobre Ciencias de la Salud, Base de Datos de Enfermería y Biblioteca Nacional de Medicina de los Estados Unidos, 2016 a 2021. Se utilizaron los siguientes Descriptores de Ciencias de la Salud y sus combinaciones en portugués e inglés: "educação em saúde" Y "atenção primária" Y "gestantes"; "educación sanitaria" y "atención primaria" y "embarazo". La muestra final estaba formada por 20 artículos. Resultados: Se encontró que el periodo de mayor acercamiento fue en el periodo de gestación contemplando el 75\% de los artículos. Por último, el 75\% de los artículos estudiados estaban relacionados con las prácticas educativas, de las cuales el diálogo era el más destacado, con un $25 \%$. Sin embargo, los talleres educativos también destacaron con un 20\%. Conclusión: Es notoria la importancia del abordaje de la educación en salud en la atención primaria para la cualificación de la salud de la gestante y de la puérpera atribuida a la prevención de agravios, enfermedades y complicaciones en este período. Así, se pone de manifiesto la necesidad de continuidad, intensificación y ampliación de las estrategias de educación sanitaria desarrolladas en atención primaria como herramienta fundamental en el ciclo gravídico-puerperal.

Palabras clave: Ciclo de embarazo-puerperal; Educación en salud; Atención Primaria.

\section{Introdução}

O período gravídico puerperal compreende um momento marcado por inúmeras modificações no aspecto psicossocial, físico e hormonal da mulher, envolvendo o surgimento de distintos sentimentos e emoções, além de dificuldades ao longo dessa etapa de vida da mulher. As alterações no organismo materno são variáveis, complexas e únicas para cada mulher, marcado por experiências e vivências diferentes a cada etapa. Alegria, medo, dúvidas, ansiedade, satisfação, insegurança são sentimentos comuns nesse período (Leite, Rodrigues, Sousa, Melo \& Fialho, 2014).

Nesse contexto, o Ministério da Saúde elaborou no início na década de 80, o Programa de Atenção Integral à Saúde da Mulher (PAISM) que propõe, a partir de seus princípios e diretrizes, a inclusão de ações educativas, preventivas, de diagnóstico, tratamento e recuperação como novas atitudes visando o desenvolvimento na qualidade da assistência à saúde da mulher (Brasil, 2016).

As ações educativas voltadas para atenção à mulher no ciclo gravídico puerperal consistem em instrumentos utilizados pelos profissionais de saúde, como forma de incluir a participação das gestantes quanto às modificações compreendidas em todas as etapas desse período, objetivando autonomia e confiança para enfrentar as diversas situações da gestação e puerpério. Ao considerar que as intervenções educativas desenvolvidas, principalmente no período do pré-natal, potencializam a resolutividade e prevenção de possíveis dúvidas e complicações advindas de falta de preparo e esclarecimento das modificações do organismo materno. Desse modo, o aprendizado nesse período orienta e prepara as gestantes com adoção de mudança com novas práticas, hábitos e atitudes resultando em um puerpério satisfatório (Guerreiro, Rodrigues, Queiroz \& Ferreira, 2014).

Em 1994, o Ministério da Saúde implantou o Programa de Saúde da Família (PSF), um modelo assistencial para estruturar a atenção primária e substituir o modelo biomédico, centrado na doença, resgatando valores e com alto potencial de resolutividade, por sua articulação com os demais níveis de complexidade assistencial (Brasil, 1997).

A Atenção Primaria à Saúde (APS), ao se tratar da principal porta de entrada do Sistema Único de Saúde (SUS) e do centro de comunicação com toda a Rede de atenção dos SUS, preconiza aprimorar a qualificação da assistência com abordagem no indivíduo, família e comunidade e compromisso de prestação de assistência à saúde de forma integral, permanente e continua e também, com estabelecimento de vínculos no âmbito interdisciplinar e multiprofissional com competências necessárias para desempenhar ações educativas, de prevenção, diagnóstico, cura e reabilitação (Conselho Nacional de Secretários de Saúde, 2015).

Portanto, a abordagem do profissional de saúde na APS é essencial ao acompanhamento e assistência integral e continua à saúde da mulher, bem como durante todo o ciclo gravídico puerperal, atendendo as demandas mais frequentes das 
gestantes e puérperas e com possibilidades de atuação como educador, capacitando essas mulheres a desenvolverem dimensões de conhecimento a cerca das modificações a cerca do seu corpo.

As estratégias de educação em saúde compreendidas como um conjunto ações voltadas para compartilhamento de conhecimentos, saberes, atitudes e práticas vivenciadas, desenvolvidas por toda equipe multiprofissional ligada a Estratégia Saúde da Família (ESF) composta de profissionais capacitados para dar assistência e orientação às gestantes e puérperas durante todas as etapas nesta fase e em todo e qualquer contato entre o profissional e a paciente, tendo como objetivo de preparação e capacitação da gestante ou de grupos de gestantes para esse período envolto de dúvidas. Nesta perspectiva, a qualidade nas condutas educativas desenvolvidas na assistência integral e contínua nesse período gestacional contribui como uma forma preventiva de redução de complicações e agravos a partir da indução de reflexão e compreensão dos determinantes do processo saúde doença propiciando a solução de problemas, e assim a melhor qualidade de vida (Cardoso et al., 2019).

Pela importância da abordagem da educação em saúde desenvolvida por profissionais capacitados no ciclo gravídico puerperal, em especial no âmbito da atenção primária e sua possível relação com os desfechos favoráveis de prevenção de agravos e melhoria de qualidade de vida das gestantes e puérperas, o estudo torna-se relevante.

Desta forma, o objetivo do estudo analisar as ações de educação em saúde desenvolvidas na atenção primária para a prevenção de agravos e melhoria da qualidade de vida no ciclo gravídico puerperal.

\section{Metodologia}

Trata-se de um estudo realizado a partir de uma revisão integrativa da literatura (RIL), a qual tem como finalidade sintetizar e analisar os resultados obtidos de múltiplas pesquisas acerca da temática investigada, a qual deve ser elaborada em seis fases. Iniciou-se com a elaboração da pergunta norteadora: as ações de educação em saúde desenvolvida na Atenção Primária têm possibilitado a prevenção de agravos e melhoria da qualidade de vida no ciclo gravídico puerperal? Em seguida foi feita uma busca bibliográfica para levantamento de artigos na biblioteca Scientific Electronic Library Online (SciELO) e base de dados da Literatura Latino-Americana e do Caribe em Ciências da Saúde (LILACS), Base de Dados de Enfermagem (BDENF) e U. S. National Library of Medicine (PubMed).

Utilizaram-se os seguintes Descritores em Ciências da Saúde (DeCS) e suas combinações nas línguas portuguesa e inglesa: "educação em saúde" AND "atenção primária" AND "gestantes"; "health education" AND "primary care" AND "pregnancy".

Os critérios de inclusão utilizados para a seleção dos estudos foram: artigos originais disponíveis na íntegra e online; publicados no período de 2016 a 2021; em inglês e português; e que retratassem o assunto principal: atenção primária à saúde e educação em saúde. Os critérios exclusão foram: artigos que não respondiam aos objetivos da pesquisa e artigos repetidos foram considerados apenas uma vez.

Dessa maneira, a amostra final foi constituída por 20 artigos. O processo de identificação, pré-seleção e seleção está descrito na Figura 1. 
Figura 1: Fluxograma referente ao processo de identificação dos artigos dos artigos. Patos, PB, Brasil, 2021.

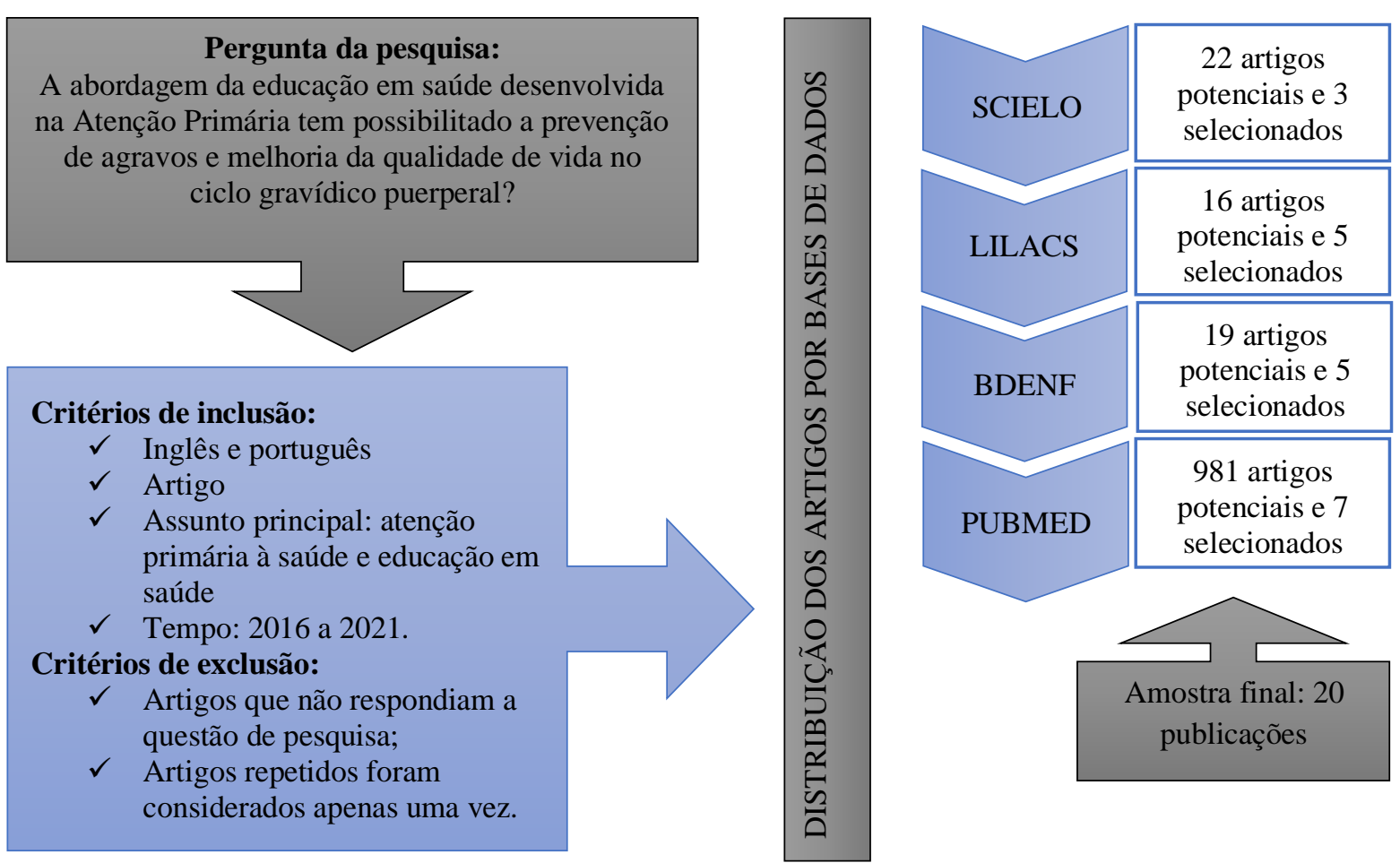

Fonte: Autoria Própria (2021).

Os dados relativos aos estudos foram sintetizados na forma de um quadro, em que foram extraídas as seguintes informações dos estudos: títulos, autores, anos, plataforma de acesso ao manuscrito, periódicos e idiomas. Ademais, os resultados das pesquisas selecionadas foram categorizados em gestação, puerpério e gestação, puerpério e quanto as atividades de educação em saúde. E nas subcategorias escuta ativa, diálogo, acolhimento humanizado, jogos e dinâmicas, programas educativos, meios de comunicação, rodas de conversa e oficinas educativas.

\section{Resultados}

O Quadro 1 expõe as características dos artigos selecionados de acordo com os quesitos autores, anos, título do artigo, periódicos e idiomas. Com relação ao periódico, pode-se observar uma variedade, mas destacaram-se Rev. enferm. UFPE on line com 20\% ( $\mathrm{n}=4)$ e BMC pregnancy and childbirth com 15\% (n=3); em relação ao idioma, destacou-se o Inglês compreendendo 65\% (n=13); e o maior número de publicações foi em 2019 com 35\% (n=7).

Quadro 1: Caracterização quanto os autores, ano, título dos artigos, periódicos e idioma de publicação.

\begin{tabular}{|l|l|l|c|}
\hline \multicolumn{1}{|c|}{ Autor(es)/ Ano } & \multicolumn{1}{|c|}{ Título do Artigo } & \multicolumn{1}{|c|}{ Periódico } \\
\hline $\begin{array}{l}\text { Marques, Tomasi, Saraiva, } \\
\text { Boing \& Geremia, 2021 }\end{array}$ & $\begin{array}{l}\text { Orientações às gestantes no pré-natal: a importância do } \\
\text { cuidado compartilhado na atenção primária em saúde }\end{array}$ & Esc. Anna Nery \\
\hline $\begin{array}{l}\text { Queiroz, Menezes, Silva, } \\
\text { Brasil \& da Silva, 2016 }\end{array}$ & $\begin{array}{l}\text { Grupo de gestantes adolescentes: contribuições para o } \\
\text { cuidado no pré-natal. }\end{array}$ & Rev. Gaúcha Enferm. \\
\hline $\begin{array}{l}\text { Rigo, Dalazen \& Garbin, } \\
2016\end{array}$ & $\begin{array}{l}\text { Impact of dental orientation given to mothers during } \\
\text { pregnancy on oral health of their children. }\end{array}$ & Einstein (São Paulo) \\
\hline
\end{tabular}




\begin{tabular}{|c|c|c|c|}
\hline $\begin{array}{l}\text { Luz, Libório, Palombo \& } \\
\text { Silva, } 2019\end{array}$ & $\begin{array}{l}\text { Núcleo de apoio à saúde da família para gestante num grupo } \\
\text { educativo: relato de experiência. }\end{array}$ & CuidArte, Enferm & Português \\
\hline Sardinha et al. (2019) & $\begin{array}{l}\text { Promoção do aleitamento materno na assistência pré-natal } \\
\text { pelo enfermeiro. }\end{array}$ & Rev. enferm. UFPE on line & Português \\
\hline Silva et al. (2019) & $\begin{array}{l}\text { Oficinas educativas com gestantes sobre boas práticas } \\
\text { obstétricas. }\end{array}$ & Rev. enferm. UFPE on line & Português \\
\hline $\begin{array}{l}\text { Quental, Nascimento, Leal, } \\
\text { Davim \& Cunha, } 2017\end{array}$ & $\begin{array}{l}\text { Práticas educativas com gestantes na atenção primária à } \\
\text { saúde. }\end{array}$ & Rev. enferm. UFPE on line & Português \\
\hline Camillo et al. (2016) & $\begin{array}{l}\text { Ações de educação em saúde na atenção primária a gestantes } \\
\text { e puérperas: revisão integrativa. }\end{array}$ & Rev. enferm. UFPE on line & Português \\
\hline $\begin{array}{l}\text { Maia, da Costa Silva \& } \\
\text { Moreira, } 2019\end{array}$ & $\begin{array}{l}\text { Eficácia de intervenções educativas com gestantes sobre o } \\
\text { grau de conhecimento em aleitamento materno. }\end{array}$ & $\begin{array}{l}\text { Revista Brasileira em } \\
\text { Promoção da Saúde }\end{array}$ & Inglês \\
\hline $\begin{array}{l}\text { Tinoco, Acioli, Neto \& } \\
\text { Silva, } 2018\end{array}$ & $\begin{array}{l}\text { Mortalidade materna e a prática educativa na atenção } \\
\text { primária à saúde. }\end{array}$ & Nursing (Säo Paulo) & Português \\
\hline Da Silva et al. (2018) & $\begin{array}{l}\text { Grupo operativo com primigestas: uma estratégia de } \\
\text { promoção à saúde. }\end{array}$ & $\begin{array}{l}\text { Revista Brasileira em } \\
\text { Promoção da Saúde }\end{array}$ & Inglês \\
\hline $\begin{array}{l}\text { Silva, Contim, Ferreira \& } \\
\text { Marqui, } 2017\end{array}$ & $\begin{array}{l}\text { Guthrie test: pregnant women's perception during prenatal } \\
\text { care. }\end{array}$ & $\begin{array}{l}\text { Revista Brasileira de Saúde } \\
\text { Materno Infantil }\end{array}$ & Inglês \\
\hline Misquita et al. (2020) & $\begin{array}{l}\text { Atendimento de gestantes na atenção primária a saúde pela } \\
\text { enfermagem durante a pandemia do SARS-COV- } 2 \text {. }\end{array}$ & Nursing (São Paulo) & Português \\
\hline Thomson et al. (2019) & $\begin{array}{l}\text { Knowledge and Education as Barriers and Facilitators to } \\
\text { Nicotine Replacement Therapy Use for Smoking Cessation in } \\
\text { Pregnancy: A Qualitative Study with Health Care } \\
\text { Professionals. }\end{array}$ & $\begin{array}{l}\text { Int J Environ Res Public } \\
\text { Health }\end{array}$ & Inglês \\
\hline $\begin{array}{l}\text { Llena, Nakdali, Sanz \& } \\
\text { Forner, } 2019\end{array}$ & $\begin{array}{l}\text { Oral Health Knowledge and Related Factors among Pregnant } \\
\text { Women Attending to a Primary Care Center in Spain. }\end{array}$ & $\begin{array}{l}\text { Int J Environ Res Public } \\
\text { Health }\end{array}$ & Inglês \\
\hline $\begin{array}{l}\text { Martín-Iglesias et al. } \\
\text { (2018) }\end{array}$ & $\begin{array}{l}\text { Effectiveness of an educational group intervention in primary } \\
\text { healthcare for continued exclusive breast-feeding: } \\
\text { PROLACT study. }\end{array}$ & $\begin{array}{l}\text { BMC Pregnancy and } \\
\text { Childbirth }\end{array}$ & Inglês \\
\hline $\begin{array}{l}\text { Paz-Pascual, } \quad \text { Artieta- } \\
\text { Pinedo \& Grandes, } 2019\end{array}$ & $\begin{array}{l}\text { Consensus on priorities in maternal education: results of } \\
\text { Delphi and nominal group technique approaches. }\end{array}$ & $\begin{array}{l}\text { BMC Pregnancy and } \\
\text { Childbirth }\end{array}$ & Inglês \\
\hline $\begin{array}{l}\text { Melo, Maia, Valente, } \\
\text { Vezzini \& Tamburlini, } \\
2018\end{array}$ & $\begin{array}{l}\text { Effectiveness of an action-oriented educational intervention } \\
\text { in ensuring long term improvement of knowledge, attitudes } \\
\text { and practices of community health workers in maternal and } \\
\text { infant health: a randomized controlled study. }\end{array}$ & BMC Med Educ & Inglês \\
\hline Malta et al. (2016) & $\begin{array}{l}\text { Educational intervention regarding diet and physical activity } \\
\text { for pregnant women: changes in knowledge and practices } \\
\text { among health professionals. }\end{array}$ & $\begin{array}{l}\text { BMC pregnancy and } \\
\text { childbirth }\end{array}$ & Inglês \\
\hline
\end{tabular}


Research, Society and Development, v. 10, n. 5, e18010514662, 2021

(CC BY 4.0) | ISSN 2525-3409 | DOI: http://dx.doi.org/10.33448/rsd-v10i5.14662

\begin{tabular}{|l|l|l|l|}
\hline $\begin{array}{l}\text { Omer, Haile, Shikur, } \\
\text { Macarayan \& Hagos, 2020 }\end{array}$ & $\begin{array}{l}\text { Effectiveness of a nutrition education and counselling } \\
\text { training package on antenatal care: a cluster randomized } \\
\text { controlled trial in Addis Ababa. }\end{array}$ & Health policy and planning & Ingles \\
\hline
\end{tabular}

Fonte: Dados de Pesquisa, 2021.

Considerando-se as plataformas de buscas, constatou-se que a base de dados de maior destaque foi a PubMed, com $35 \%(\mathrm{n}=7)$ dos artigos estudados.

Figura 2: Distribuição das plataformas de acesso aos manuscritos selecionados

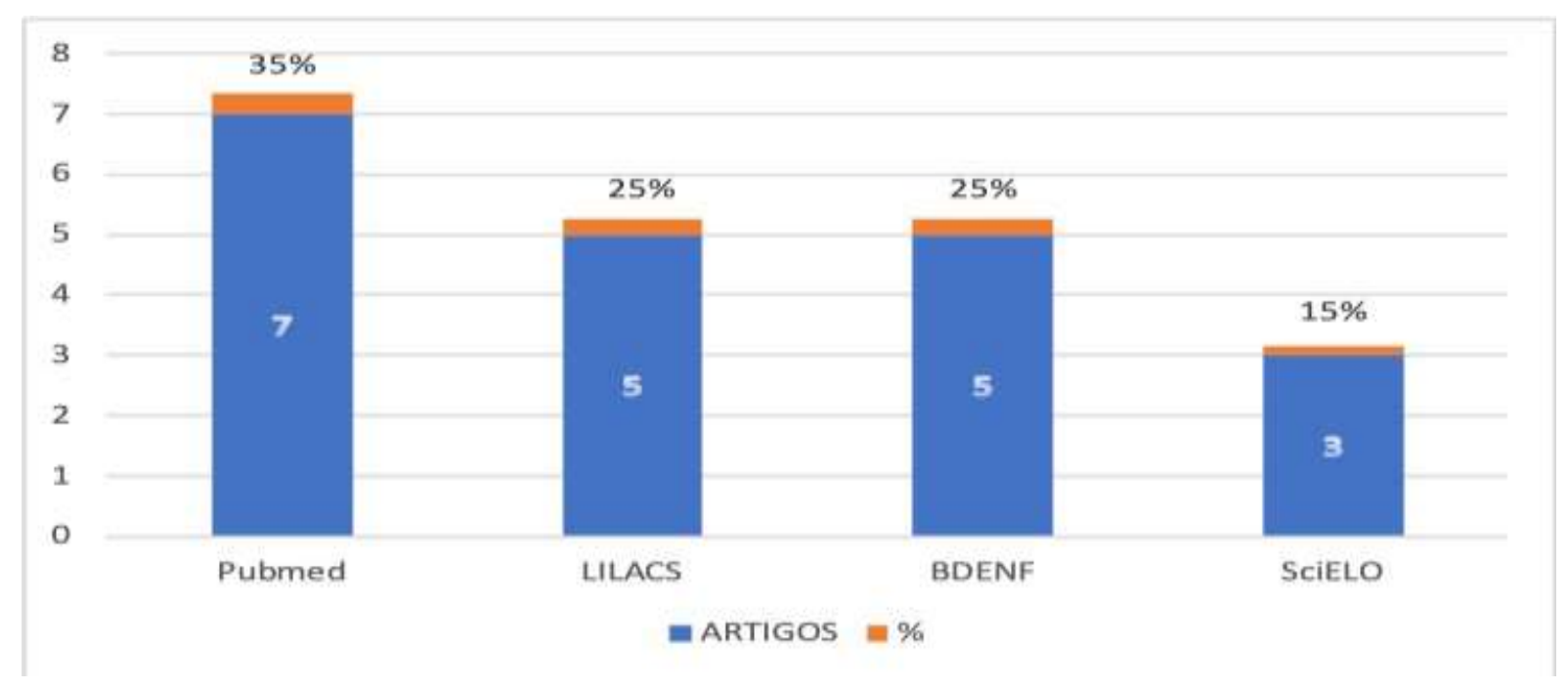

Fonte: Dados de Pesquisa (2021).

Quanto à categorização levaram-se em consideração os seguintes aspectos, o período de abordagem da educação em saúde durante o ciclo gravídico puerperal e suas finalidades. Deste modo, o maior número de atividades educativas contemplou a gestação $(75 \%$; $\mathrm{n}=15)$; seguida da categoria gestação e puerpério $(20 \% ; \mathrm{n}=4)$ e apenas puerpério $(5 \%$; $\mathrm{n}=1)$ dos artigos estudados.

Quanto às atividades de educação em saúde, os estudos majoritariamente contemplaram as práticas educativas (75\%; $\mathrm{n}=15)$. Em relação às subcategorias, destaque para o diálogo $(25 \% ; \mathrm{n}=5)$ e as oficinas educativas $(20 \% ; \mathrm{n}=4)$ (Quadro 2). 
Quadro 2: Caracterização quanto categorias, subcategorias, autores e ano, n e \%.

\begin{tabular}{|c|c|c|c|c|}
\hline Categorias & Subcategorias & Autores e ano & $\mathbf{n}$ & $\%$ \\
\hline \multirow{6}{*}{$\begin{array}{l}\text { Práticas educativas } \\
\qquad(75 \% ; \mathrm{n}=15)\end{array}$} & Escuta ativa & Camillo et al (2016) & 1 & 5 \\
\hline & Diálogo & $\begin{array}{l}\text { Malta et al (2016); Silva et al. (2017); Thomson et al (2019); } \\
\text { Misquita et al. (2020); Sardinha et al. (2019). }\end{array}$ & 5 & 25 \\
\hline & Acolhimento humanizado & $\begin{array}{c}\text { Tinoco (2018); Marques et al (2021); Rigo, Dalazen \& } \\
\text { Garbin, 2016; Llena et al. (2019) }\end{array}$ & 4 & 20 \\
\hline & Jogos e dinâmicas & Quental et al. (2017) & 1 & 5 \\
\hline & Programas educativos & Paz-Pascual, Artieta-Pinedo \& Grandes, 2019 & 1 & 5 \\
\hline & $\begin{array}{l}\text { Meios de comunicação (Palestra, } \\
\text { Panfleto, Cartaz e Vídeo) }\end{array}$ & $\begin{array}{l}\text { Maia, da Costa Silva \& Moreira, 2019; Melo et al. (2018); } \\
\text { Omer et al. (2020) }\end{array}$ & 3 & 15 \\
\hline \multirow{2}{*}{$\begin{array}{l}\text { Grupo de gestantes } \\
\qquad(25 \% ; \mathrm{n}=5)\end{array}$} & Rodas de conversa & Queiroz et al (2016) & 1 & 5 \\
\hline & Oficinas educativas & $\begin{array}{c}\text { Silva et al (2019); Luz et al (2019); Da Silva et al. (2018); } \\
\text { Martín-Iglesias et al. (2018) }\end{array}$ & 4 & 20 \\
\hline
\end{tabular}

Fonte: Dados de Pesquisa (2021).

\section{Discussão}

As ações educativas no período gravídico puerperal propiciam a prevenção e promoção de saúde, possibilitando o desenvolvimento de autonomia, confiança e preparação da mulher pelo acesso aos conhecimentos, esclarecimentos, atitudes, informações e mudanças de comportamentos, tornando-a sujeito ativo no seu processo saúde-doença. Sendo assim, os profissionais de saúde devidamente capacitados podem ser instrumentos importantes no processo de compartilhamento de experiências, práticas e saberes qualificando a atenção à saúde no ciclo gravídico puerperal no contexto da atenção primária (Camillo et al., 2016).

A educação em saúde acontece principalmente através do desenvolvimento de práticas educativas voltadas para escuta e conversa, acolhimento, diálogo, jogos e dinâmicas entre gestantes e profissionais de saúde, de modo que o processo de educação em saúde torna-se um determinante essencial para o empoderamento da mulher acerca do seu processo saúde doença. Nessa perspectiva, é importante ressaltar que as práticas educativas em saúde têm um grande potencial transformador de construção do saber que repercute significativamente no desenvolvimento satisfatório e saudável da gestação e puerpério, proporcionam aprendizado, conhecimento, troca de experiências e vivências, esclarecimento de dúvidas e em especial, integração entre os componentes inseridos nesse processo, profissionais, usuários e familiares (Quental et al., 2017).

Destaca-se que a maioria das gestantes apresentam dúvidas acerca do processo gestacional e puerpério, além de acreditarem em crenças e mitos existentes o que dificultam adoção de práticas e hábitos satisfatórios. Desse modo, o uso de dinâmicas educativas sobre mitos e verdades a cerca desse momento da vida da mulher proporciona conhecimento e assim, desmitificação. Dessa maneira, ressalta a importância da disseminação de informação através de trocas de saberes, diálogo e escuta qualificada entre as gestantes e os profissionais de saúde para adesão às novas práticas, em especial, ao aleitamento materno exclusivo (Sardinha et al., 2019).

Segundo o Ministério da Saúde (2019), na análise epidemiológica do Brasil da última década, o país mostrou redução importante nos indicadores de mortalidade materna e infantil, embora permanecendo com valor acima dos parâmetros sugeridos pela Organização Mundial de Saúde e assim, não atingindo ainda a meta desejada.

Esses indicadores refletem a qualidade assistencial à saúde no ciclo gravídico puerperal e proporcionam informações necessárias para a prevenção da mortalidade materna no contexto da atenção primária. Os dados auxiliam os profissionais e 
gestores envolvidos na organização do serviço de saúde da atenção primária com implementação de novas estratégias educativas que aumentem a assistência à saúde da mulher (Tinoco et al., 2018).

De modo particular, a investigação indica que a temática da educação em saúde durante o período de gestação e puerpério parece pouco abordada no país, especialmente pela insatisfação diante da insuficiência de abordagem de práticas educativas no contexto do período gravídico puerperal, especialmente nas consultas de pré-natal, que por sua vez, apresenta-se como um momento oportuno para por em prática as ações educativas (Camillo et al., 2016; Quental et al., 2017).

Como exemplo de estratégias educativas na atenção primária, destacaram-se os grupos de gestantes, os quais atuam como um espaço de diálogo e comunicação entre as gestantes e entre elas e os profissionais de saúde, permitindo o acesso e construção do conhecimento, aquisição de habilidades e mudanças de comportamentos, o que as torna protagonistas ativas do seu processo saúde doença, além de contribuir com o estabelecimento de vínculos e também continuidade de assistência ressaltando a humanização (Martín-Iglesias et al., 2018; Luz et al., 2019).

Compartilham-se experiências e percepções individuais e pessoais, além de conhecimentos e saberes a cerca do processo de modificações do organismo materno, sobretudo também a cerca dos diretos durante todo o ciclo gravídico puerperal, levando a construção de autonomia e autoconfiança da gestante (Silva et al., 2019).

Os grupos educativos são fundamentais, uma vez que através do processo de conversa e escuta qualificada possibilita o esclarecimento de dúvidas, queixas comuns, desmistificação de crenças e tabus, orientações e, sobretudo, redução de medos, angústias e ansiedades inerentes ao processo gestacional, atendendo as necessidades e particularidades do grupo de gestantes atendidas (Queiroz et al., 2016; Da Silva et al., 2018).

As orientações no pré-natal abordadas na atenção primária devem começar a partir da primeira consulta de pré-natal com o propósito de estabelecimento de vínculos e continuidade do atendimento nas próximas consultas, além de necessárias para preparar a mulher para todo o período gestacional e após o nascimento, o puerpério, possibilitando uma gestação saudável.

Diante que, o número de consultas estaria relacionado com a satisfação da adequação das orientações prestadas na assistência, de modo que orientações realizadas conjuntamente pelos profissionais da equipe da atenção primária que exercem o papel de educador, médicos e enfermeiros refletem em uma maior adequação das orientações e então, na melhoria da qualidade de vida da mulher. Sendo a orientação que mais se destacou foi sobre os sinais de riscos na gestação (Marques et al., 2021).

Como exemplo, a orientação odontológica sobre a saúde bucal recebida durante o pré-natal influencia na maior percepção das mulheres sobre saúde bucal, desta forma possibilita a adoção de novas atitudes e comportamentos advindos aos conhecimentos adquiridos, especialmente quanto ao autocuidado e prevenção, o que reflete positivamente sobre a qualidade de vida e assistência à saúde da mulher (Rigo, Dalazen \& Garbin, 2016; Llena et al., 2019).

Por fim, observaram-se lacunas ou falhas nas orientações de autocuidado e autonomia da mulher (Silva et al., 2017; Marques et al., 2021). De acordo com os autores, alguns fatores podem estar associados ao desencorajamento e falta de adesão ao aleitamento materno, tais como insegurança, medo, receios advindos de falta de informação, acarretando em desmame precoce, baixo peso e adoecimento da criança.

As ações educativas voltadas para o aleitamento materno colaboram com a adesão do ato da amamentação (Sardinha et al., 2019). De modo que, verifica-se que as estratégias educativas são responsáveis por melhorar os níveis de conhecimentos das gestantes a cerca da temática, sendo imprescindível para o empoderamento da mulher no processo fisiológico e prolongamento do aleitamento materno exclusivo (Maia, da Costa Silva \& Moreira, 2019). No mais, as evidências frisam que 
quando a gestante recebe orientações e aconselhamento quanto à prática da amamentação, os resultados refletem no aumento significativo de mulheres que aderem ao aleitamento materno exclusivo (Martín-Iglesias et al., 2018).

Estudo objetivando, relacionar a educação nutricional e a relação com melhoria de adoções de práticas alimentares satisfatórias por mulheres grávidas, mostram resultados que refletem positivamente quando realizado por profissionais devidamente capacitados e treinados com habilidades de aconselhamento quanto aos dos cuidados nutricionais, além de refletir no engajamento das gestantes e dos profissionais durante as consultas e na compreensão com adoção de novos comportamentos, atitudes e práticas (Omer et al., 2020).

Corroboram com a afirmativa, Malta et al. (2016), ao avaliarem os efeitos de uma intervenção educativa focada na melhoria dos conhecimentos e práticas dos profissionais de saúde a cerca da temática de alimentação e atividade física durante a gestação, uma vez os profissionais são instrumentos essenciais exercendo o papel de educador ao transmitir conhecimentos e práticas, especialmente no período de pré-natal em que o vínculo e contato são mais intensos. Quanto aos resultados, as gestantes atendidas por profissionais detentor do conhecimento apresentam uma probabilidade maior em receber orientações sobre a temática, em detrimento as não atendidas por esses profissionais, acarretando em uma maior adoção dessas práticas por gestantes orientadas e aconselhadas durante as consultas de pré-natal.

É importante ressaltar a escuta e conversa entre a gestante/puérpera e o profissional de saúde, através desse diálogo pode-se enfatizar pontos importantes e necessários durante o curso da gestação e puerpério, além de esclarecer dúvidas advindas do processo e favorecer o cuidado e acolhimento humanizado com adoção de novas práticas e hábitos (Camillo et al., 2016).

Diante do exposto, é importante frisar que para o desenvolvimento da promoção em a saúde através da educação em saúde, é essencial o conhecimentos a cerca de recomendações, diretrizes atuais, além de treinamentos e capacitações por parte dos prestadores de cuidados, a fim de desempenharem o seu papel como educador na assistência às gestantes de modo seguro e relevante, ao aconselhar, orientar, apoiar, transmitir conhecimentos e práticas, tornando-a sujeito ativo nesse processo e motivada para aderir a novos comportamentos, atitudes e práticas que iram refletir em resultados positivos durante o ciclo gravídico puerperal (Melo et al., 2018).

Sobre essa perspectiva, Misquita et al. (2020) ressaltam a importância do papel do profissional de saúde como educador diante o enfrentamento da pandemia causada pelo SARS-Cov 2, e ressalta a necessidade de está capacitado e apto para orientar e propagar as medidas preventivas para os cuidados das gestantes, uma vez que estas se enquadram no grupo de risco e precisam frequentar as unidades de atenção primária para o acompanhamento do pré-natal de maneira contínua.

Contemplando a assertiva, a educação em saúde no ciclo gravídico puerperal deve contemplar temas voltados para as necessidades das gestantes e puérperas, a fim de atingir resultados satisfatórios com conhecimentos úteis, para refletir em mudança de comportamento e consequentemente, melhoria da qualidade de vida da glestante (Paz-Pascual, Artieta-Pinedo \& Grandes, 2019).

Condizente ao exposto, para Thomson et al. (2019), a educação em saúde é um instrumento imprescindível para quebrar barreirar e facilitar o alcance do sucesso na cessação do tabagismo em mulheres grávidas a partir do incentivo ao uso de Terapia de Reposição de Nicotina (TRN), visto que, o tabagismo na gestação é prejudicial e uma das causas de complicações como exemplo de aborto, natimorto e nascimentos prematuros, além da sua relação com complicações de saúde na infância.

Essa revisão integrativa apresenta certas limitações diante à escassez de estudos que abordem a temática a cerca da abordagem da educação em saúde desenvolvida na atenção primária. Por se tratar de um tema relevante apresentou limitações também, especialmente para encontrar trabalhos que abordassem o manejo das estratégias e práticas educativas no contexto da atenção primária para gestantes e puérperas. Além disso, outra limitação válida é das lacunas existentes diante a atuação dos 
variados profissionais em relação à adequação e qualificação das orientações ofertadas durante a assistência no ciclo gravídico puerperal.

\section{Conclusão}

De acordo com os dados analisados na literatura, a presente revisão evidenciou a necessidade da abordagem da educação em saúde instituída na atenção primária no manejo do cuidado a gestante e a puérpera, as quais podem contribuir com a prevenção e redução de agravos, doenças e complicações, e estão estreitamente ligadas ao progresso dos níveis de qualidade de vida no ciclo gravídico puerperal.

As ações que estão sendo desenvolvidas centradas na promoção de saúde contemplam dinâmica de conhecimento, esclarecimento de dúvidas, conscientização e informação, e também estabelecimento de vínculo de confiança a partir de diálogo e interação entre a equipe multiprofissional. As estratégias educativas reduzem os anseios e dúvidas decorrentes do processo gestacional, e mostram desfechos satisfatórios na prevenção de agravos no período gestacional e no pós-parto, propiciando melhor qualificação na atenção à saúde da mulher.

Diante a relevância da temática, sugere-se o incentivo contínuo da adoção de estratégias educativas na Atenção Básica, especialmente no período gravídico e puerperal. Ainda, é importante a publicização de relatos de experiência referentes às práticas referentes ao objeto de estudo e o desfecho de novas pesquisas na área, em face da relevância da temática.

\section{Referências}

Brasil. Ministério da Saúde. Secretaria de Assistência a Saúde. (1997). Saúde da Família: uma estratégia para a reorientação do modelo assistencial. Brasil. Ministério da Saúde.

Brasil. Ministério da Saúde (2016). Saúde das mulheres. Brasília: Ministério da Saúde.

Camillo, B. S., Nietsche, E. A., Salbego, C., Cassenote, L. G., Osto, D., Silva, D., \& Böck, A. (2016). Ações de educação em saúde na atenção primária a gestantes e puérperas: revisão integrativa. Rev. enferm. UFPE on line, 4894-4901.

Cardoso, S. L., de Souza, M. E. V., Oliveira, R. S., Souza, A. F., Lacerda, M. D. D. F., Oliveira, N. T. C., ... \& Medeiros, K. M. F. (2019). Ações de promoção para saúde da gestante com ênfase no pré-natal. Revista Interfaces: Saúde, Humanas e Tecnologia, 7(1), $180-186$.

Conselho Nacional de Secretários de Saúde. (2015). A atenção primária e as redes de atenção à saúde. Brasília: CONASS.

Da Silva, M. A. M., Marques, F. M., Brito, M. D. C. C., Viana, R. S., Mesquita, A. L. M., Silva, A. S. R., \& Gomes, L. C. (2018). Grupo ope rativo com primigestas: uma estratégia de promoção à saúde. Revista Brasileira em Promoção da Saúde, 31(1).

Guerreiro, E. M., Rodrigues, D. P., Queiroz, A. B. A., \& Ferreira, M. D. A. (2014). Educação em saúde no ciclo gravídico-puerperal: sentidos atribuídos por puérperas. Revista brasileira de enfermagem, 67(1), 13-21.

Leite, M. G., Rodrigues, D. P., Sousa, A. A. S. D., Melo, L. P. T. D., \& Fialho, A. V. D. M. (2014). Sentimentos advindos da maternidade: revelações de um grupo de gestantes. Psicologia em estudo, 19(1), 115-124.

Llena, C., Nakdali, T., Sanz, J. L., \& Forner, L. (2019). Oral Health Knowledge and Related Factors among Pregnant Women Attending to a Primary Care Center in Spain. International journal of environmental research and public health, 16(24), 5049.

Luz, C. A. S., Libório, R., Palombo, C. N. T., \& Silva, J. C. D. (2019). Núcleo de apoio à saúde da família para gestante num grupo educativo: relato de experiência. CuidArte, Enferm, 199-203.

Maia, A. K., da Costa Silva, B. Y., \& Moreira, L. C. J. (2019). Eficácia de intervenções educativas com gestantes sobre o grau de conhecimento em aleitamento materno. Revista Brasileira em Promoção da Saúde, 32.

Malta, M. B., Carvalhaes, M. A. D. B. L., Takito, M. Y., Tonete, V. L. P., Barros, A. J., de Lima Parada, C. M. G., \& Benício, M. H. D. A. (2016). Educational intervention regarding diet and physical activity for pregnant women: changes in knowledge and practices among health professionals. $B M C$ pregnancy and childbirth, 16(1), 1-9.

Marques, B. L., Tomasi, Y. T., Saraiva, S. D. S., Boing, A. F., \& Geremia, D. S. (2021). Orientações às gestantes no pré-natal: a importância do cuidado compartilhado na atenção primária em saúde. Escola Anna Nery, 25(1).

Martín-Iglesias, S., Santamaría-Martín, M. J., Alonso-Álvarez, A., Rico-Blázquez, M., del Cura-González, I., Rodríguez-Barrientosn, R., ... \& Villa-Arranz, M. (2018). Effectiveness of an educational group intervention in primary healthcare for continued exclusive breast-feeding: PROLACT study. BMC pregnancy and childbirth, 18(1), 1-10. 
Research, Society and Development, v. 10, n. 5, e18010514662, 2021

(CC BY 4.0) | ISSN 2525-3409 | DOI: http://dx.doi.org/10.33448/rsd-v10i5.14662

Melo, T. E. L., Maia, P. F. C. M. D., Valente, E. P., Vezzini, F., \& Tamburlini, G. (2018). Effectiveness of an action-oriented educational intervention in ensuring long term improvement of knowledge, attitudes and practices of community health workers in maternal and infant health: a randomized controlled study. BMC medical education, 18(1), 224-224.

Misquita, M. S., da Silva, P. G., de Abreu Braz, G., Sousa, A. B. D. A. G., Melo, D. F. C., \& de Paula Melo, F. N. (2020). Atendimento de gestantes na atenção primária a saúde pela enfermagem durante a pandemia do SARS-COV-2. Nursing (São Paulo), 23(269), 4723-4730.

Paz-Pascual, C., Artieta-Pinedo, I., \& Grandes, G. (2019). Consensus on priorities in maternal education: results of Delphi and nominal group technique approaches. BMC pregnancy and childbirth, 19(1), 1-13.

Queiroz, M. V. O., Menezes, G. M. D., Silva, T. J. P., Brasil, E. G. M., \& da Silva, R. M. (2016). Grupo de gestantes adolescentes: contribuições para o cuidado no pré-natal. Revista Gaúcha de Enfermagem, 37.

Quental, L. L. C., Nascimento, L. C. C. D. C., Leal, L. C., Davim, R. M. B., \& Cunha, I. C. B. C. (2017). Práticas educativas com gestantes na atenção primária à saúde. Rev. enferm. UFPE on line, 5370-5381.

Rigo, L., Dalazen, J., \& Garbin, R. R. (2016). Impact of dental orientation given to mothers during pregnancy on oral health of their children. Einstein (São Paulo), 14(2), 219-225.

Sardinha, D. M., Maciel, D. O., Gouveia, S. C., Pamplona, F. C., Sardinha, L. M., Carvalho, M. D. S. B. D., \& Silva, A. G. I. D. (2019). Promoção do aleitamento materno na assistência pré-natal pelo enfermeiro. Rev. enferm. UFPE on line, 852-857.

Silva, J. C. B. D., Lima, R. M. C., Lins, M. A. R. A., Lemos, M. E. P., Carvalho, M. V. G. D., \& Silva, S. V. (2019). Oficinas educativas com gestantes sobre boas práticas obstétricas. Rev. enferm. UFPE on line, 255-260.

Silva, M. P. C., Contim, D., Ferreira, L. A., \& Marqui, A. B. T. D. (2017). Guthrie test: pregnant women's perception during prenatal care. Revista Brasileira de Saúde Materno Infantil, 17(2), 291-298.

Tinoco, T. F., Acioli, S., Neto, M., \& Silva, L. A. (2018). Mortalidade materna e a prática educativa na atenção primária à saúde. Nursing (Säo Paulo), 25352541 .

Thomson, R., McDaid, L., Emery, J., Naughton, F., Cooper, S., Dyas, J., \& Coleman, T. (2019). Knowledge and education as barriers and facilitators to nicotine replacement therapy use for smoking cessation in pregnancy: a qualitative study with health care professionals. International journal of environmental research and public health, 16(10), 1814.

Omer, A. M., Haile, D., Shikur, B., Macarayan, E. R., \& Hagos, S. (2020). Effectiveness of a nutrition education and counselling training package on antenatal care: a cluster randomized controlled trial in Addis Ababa. Health Policy and Planning, 35(Supplement_1), i65-i75. 